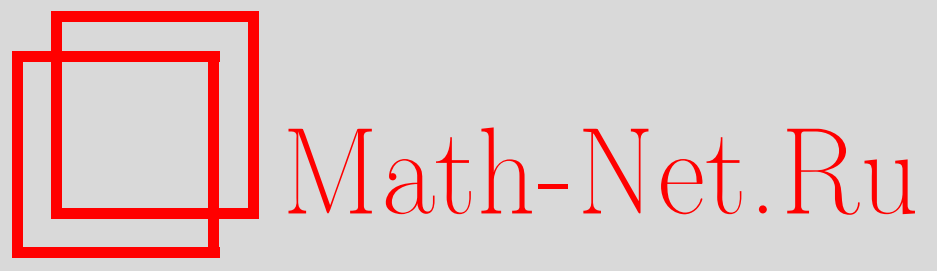

А. А. Вороненко, О методе разложения для распознавания принадлежности инвариантным классам, Дискрет. матем., 2002, том 14, выпуск 4, 110-116

DOI: https://doi.org/10.4213/dm266

Использование Общероссийского математического портала Math-Net.Ru подразумевает, что вы прочитали и согласны с пользовательским соглашением http://www . mathnet.ru/rus/agreement

Параметры загрузки:

IP : 3.93.64.190

26 апреля 2023 г., 08:13:28

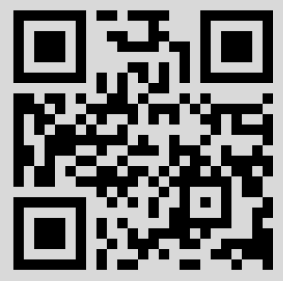


УдК 519.7

\title{
О методе разложения для распознавания принадлежности инвариантным классам
}

\author{
() 2002 г. А. А. Вороненко
}

В работе строится общий метод распознавания принадлежности функций классам, замкнутым относительно подстановки констант, использующий разложение по переменной.

\section{1. Введение}

Известны различные постановки задачи распознавания свойств дискретных функций. В настоящей работе распознавание ведется схемами из функциональных элементов в произвольном полном базисе (СФЭ), входом которых является вектор-столбец значений функция, а выходом - бит, равный 1 для функщий, обладающих заданным свойством, 0 в противном случае.

Следующая теорема является основной.

Теорема 1. Пусть F - произвольный класс булевых функций, замкнутый относительно подстановки констант на место переменных, и пусть существуют кодирование функиий, принадлежащих $F$, и последовательность схем $\left\{\Sigma_{m}\right\}$, обладающие следуюшими свойствами:

(1) кодом константы является она сама;

(2) если на вход произвольной схемы $\Sigma_{m}$ подать коды подфункций

$$
f\left(x_{1}, \ldots, x_{m-1}, 0\right), \quad f\left(x_{1}, \ldots, x_{m-1}, 1\right),
$$

принадлежащих $F$, то схема $\Sigma_{m}$ распознает принадлежность функиии $f\left(x_{1}, \ldots, x_{m}\right)$ классу $F$;

(3) если функция $f\left(x_{1}, \ldots, x_{m}\right)$ принадлежит классу $F$, то выход схемы $\Sigma_{m}$ содержит код функиии $f\left(x_{1}, \ldots, x_{m}\right)$;

(4) сложность схемы $\Sigma_{m}$ не превосходит $\beta(m)$.

Тогда существует схема $S_{n}$, распознающая принадлежность классу $F$ произвольной функци п переменных, заданной векторно, сложности, не больщей

$$
\left(2^{n}-2\right) L\left(x_{1} \& x_{2}\right)+\sum_{k=1}^{n} 2^{n-k} \beta(k),
$$

где $L\left(x_{1} \& x_{2}\right)$ - сложность реализачии конъюнкции двух переменных. 
Доказательство. Схема $S_{n}$ будет иметь $n$ уровней. На $k$-м уровне расположены $2^{n-k}$ подсхем, строящих по кодам подфункций

$$
f\left(x_{1}, \ldots, x_{k-1}, 0, \sigma_{k+1}, \ldots, \sigma_{n}\right), \quad f\left(x_{1}, \ldots, x_{k-1}, 1, \sigma_{k+1}, \ldots, \sigma_{n}\right)
$$

код подфункции $f\left(x_{1}, \ldots, x_{k}, \sigma_{k+1}, \ldots, \sigma_{n}\right)$ и проверяющих принадлежность классу $F$ получаемых подфункций. Сложность каждой подсхемы на $k$-м уровне не превосходит $\beta(k)$. Выходом схемы $S_{n}$ является конъюнкция выходов всех подсхем, проверяющих принадлежность классу $F$ всех промежуточных подфункций. Сложность всей схемы не будет превосходить

$$
\left(2^{n}-2\right) L\left(x_{1} \& x_{2}\right)+\sum_{k=1}^{n} 2^{n-k} \beta(k)
$$

Теорема доказана.

Следствие 1. Если ряд $\sum_{k=1}^{\infty} \beta(k) 2^{-k}$ сходится, то принадлежность инвариантному классу распознается с линейной сложностью относительно длины $2^{n}$ вектор-столбиа булевой функции.

Для класса монотонных функций конструкция теоремы 1 изложена в работе [1]. В настоящей работе этот результат обобщен для классов частичных монотонных функщий и поляризуемых функщий. Теорема 1 применяется для получения схем линейной сложности, распознающих свойство доопределимости до линейной функции. Напомним способ кодирования монотонных функций, использованный в [1].

Рассмотрим разбиение на цепи Анселя. Напомним, что цепью называется возрастающая последовательность соседних наборов. Разбиение на цепи Анселя строится по индукции. 0-мерные подкубы $m$-мерного булева куба, наборы $\left(\sigma_{1}, \ldots, \sigma_{m}\right)$, покрываются одной цепью, состоящей из этого набора. Пусть $k<m$ и $k$-мерные подкубы, множества наборов вида $\left(x_{1}, \ldots, x_{k}, \sigma_{k+1}, \ldots, \sigma_{m}\right)$ разбиты на цепи. Назовем цепи, наборы которых отличаются лишь $(k+1)$-й координатой, параллельными. Разбиение $(k+1)$-мерных подкубов получается передачей всем цепям с нулевой $(k+1)$-й координатой наибольших наборов из параллельных им цепей. После этого цепи, не содержащие наборов, из дальнейшего рассмотрения исключаются. Количество цепей Анселя, покрывающих булев куб размерности $m$, равно (см. [2])

$$
\left(\begin{array}{c}
m \\
{[m / 2]}
\end{array}\right)=O\left(m^{-1 / 2} 2^{m}\right) .
$$

В [1] в качестве кода произвольной монотонной булевой функции использовался упорядоченный набор чисел, равных количеству нулей на цепях Анселя. Длина кода одинакова для всех монотонных функций $m$ переменных и есть

$$
O\left(m^{-1 / 2} 2^{m} \log (m+1)\right) .
$$

\section{2. Частичные монотонные функции}

Каждое значение частичной булевой функщии может быть закодировано двумя битами. Не всюду определенная булева функщия называется частичной монотонной, если для 
нее существует монотонное доопределение. Замыканием частичной монотонной функщии $f(x)$ называется функщия $\tilde{f}(x)$, задаваемая соотношениями

$$
\tilde{f}(x)= \begin{cases}1, & \text { если } \exists y \leqslant x \mid f(y)=1, \\ 0, & \text { если } \exists y \geqslant x \mid f(y)=0 \\ f(x) & \text { не определена в противном случае. }\end{cases}
$$

Кодом частичной монотонной булевой функции назовем упорядоченный набор троек чисел, состоящих из числа нулей $\left(n_{0}\right)$, единищ $\left(n_{1}\right)$ и общего количества наборов (length) ее монотонного замыкания на цепях Анселя. Длина кода одинакова для всех частичных монотонных функций $m$ переменных и есть $O\left(m^{-1 / 2} 2^{m} \log (m+1)\right)$.

Замечание 1. Все рассматриваемые ниже классы функций замкнуты относительно подстановки констант на место переменных. Для частичных монотонных функций удобно следующее кодирование констант: 00 - неопределенность, 10 - ноль, 01 - единица. Тогда первый бит кода равен $n_{0}$, второй $n_{1}$, а параметр length $=1$.

Кроме этого все используемые кодирования констант, функщий, зависящих от нуля переменных, получаются из вектор-столбца схемами константной сложности.

Опишем схему $\Sigma_{m}^{\text {part }}$. Схема $\Sigma_{m}^{\text {part }}$ состоит из $\left(\begin{array}{c}m-1 \\ {[(m-1) / 2]}\end{array}\right)$ подсхем, входами которых являются пары троек, соответствующие параллельным цепям Анселя. Выход каждой подсхемы состоит из контрольного бита (mon) и кодов двух новых цепей (или одной, если цепи содержали по одному набору) в кубе размерности $m$. Подсхемы схемы $\Sigma_{m}^{\text {part }}$ осуществляют следующие преобразования:

$$
\begin{aligned}
\operatorname{mon} & =n_{1}^{0}+n_{0}^{1} \leqslant \text { length }, \\
\max _{0} & =\max \left\{n_{0}^{0}, n_{0}^{1}\right\}, \\
\max _{1} & =\max \left\{n_{1}^{0}, n_{1}^{1}\right\}, \\
\text { length }^{l} & =\text { length }+1, \\
n_{0}^{l} & =\max _{0}+\left(n_{0}^{1}=\text { length }\right), \\
n_{1}^{l} & =\max _{1}+\left(n_{1}^{1}>0\right), \\
\text { length } & =\text { length }-1, \\
n_{0}^{s} & =n_{0}^{1}-\left(n_{0}^{1}=\text { length }\right), \\
n_{1}^{s} & =n_{1}^{1}-\left(n_{1}^{1}>0\right) .
\end{aligned}
$$

Здесь $l$ - удлиненная новая цепь, $s$ - укороченная.

Выходом схемы $\Sigma_{m}^{\text {part }}$ является конъюнкция контрольных битов и набор кодов новых цепей Анселя.

Лемма 1. Пусть $f_{0}=f\left(x_{1}, \ldots, x_{m-1}, 0\right) u f_{1}=f\left(x_{1}, \ldots, x_{m-1}, 1\right)$ - частичные монотонные булевы функции. Тогда СФЭ $\Sigma_{m}^{\text {part }}$ удовлетворяет условиям теоремы 1 и имеет сложность

$$
\beta(m)=O\left(m^{-1 / 2} 2^{m} \log (m+1)\right) .
$$

Доказательство. Если частичная булева функция немонотонна, то существуют два набора, на которых нарушается монотонность. В силу частичной монотонности $f_{0}$ и $f_{1}$ эти наборы отличаются в $m$-й координате. Соединим эти наборы произвольной цепью 
возрастающих наборов. По определению монотонного замыкания на соседних по $m$-й координате наборах $\alpha \leqslant \beta$ этой цепи $\tilde{f}_{0}(\alpha)=1, \tilde{f}_{1}(\beta)=0$. Поэтому функция $f$ немонотонна тогда и только тогда, когда существует цепь Анселя в $(m-1)$-мерном булевом кубе, на которой нижняя единица $\tilde{f}_{0}$ лежит выше верхнего нуля $\tilde{f}_{1}$, или $n_{1}^{0}+n_{0}^{1}>$ length. Аналогичные рассуждения показывают, что в случае частичной монотонности $f$ схема $\Sigma_{m}^{\text {part }}$ выдает код $f$. Сравнение чисел, сложение, вычитание и взятие максимума реализуются подсхемами линейной сложности. Конъюнкция результатов сравнений имеет линейную сложность относительно числа сравнений. Таким образом, схема $\Sigma_{m}^{\text {part }}$ выполняет требуемые в теореме 1 преобразования и имеет линейную относительно длины своего входа сложность. Лемма доказана.

Теорема 2. Существует последовательность схем $S_{n}$, распознающих частичную монотонность произвольной функции $n$ переменных, заданной векторно, сложности $O\left(2^{n} \sqrt{n} \log n\right)$.

Доказательство. Из теоремы 1 и леммы 1 следует существование схем, распознающих частичную монотонность $S_{n}$, сложности

$$
\left(2^{n}-2\right) L\left(x_{1} \& x_{2}\right)+\sum_{k=1}^{n} 2^{n-k} O\left(k^{-1 / 2} 2^{k} \log (k+1)\right)=O\left(2^{n} \sum_{k=1}^{n} \frac{\ln k}{\sqrt{k}}\right) .
$$

Оценим конечную сумму при помощи интеграла. Функция $\ln k / \sqrt{k}$ убывает при $k \geqslant 8$, поэтому

$$
\sum_{k=1}^{n} \frac{\ln k}{\sqrt{k}} \leqslant \sum_{k=1}^{8} \frac{\ln k}{\sqrt{k}}+\int_{8}^{n} \frac{\ln x}{\sqrt{x}} d x .
$$

Первое слагаемое является константой, а

$$
\int_{8}^{n} \frac{\ln x}{\sqrt{x}} d x=\left.(2 \sqrt{x} \ln x-4 \sqrt{x})\right|_{8} ^{n}=O(\sqrt{n} \log n) .
$$

Теорема доказана.

\section{3. Поляризуемые функции}

Пусть для функщия $f\left(x_{1}, \ldots, x_{n}\right)$ существует вектор $\beta$ такой, что функщия $f\left(x_{1}^{\beta_{1}}, \ldots, x_{n}^{\beta_{n}}\right)$ монотонна. Тогда функция $f\left(x_{1}, \ldots, x_{n}\right)$ называется поляризуемой, а трехзначный набор $\beta$, определяемый соотношениями

$$
\beta_{i}= \begin{cases}1, & \text { если } f(x) \text { монотонна по } x_{i}, \\ 0, & \text { если } f(x) \text { антимонотонна по } x_{i}, \\ -, & \text { если переменная } x_{i} \text { несущественна для } f(x),\end{cases}
$$

называется вектором поляризации. Задача вероятностного тестирования свойств монотонности и поляризуемости рассматривалась в [3]. Кодом поляризуемой булевой функщи назовем упорядоченный набор чисел, состоящий из вектора поляризации $\beta$ и кода монотонной функщии $f\left(x_{1}^{\beta_{1}}, \ldots, x_{m}^{\beta_{m}}\right)$. Длина кода также одинакова для всех поляризованных функций $m$ переменных и есть $O\left(m^{-1 / 2} 2^{m} \log (m+1)\right)$. 
Опишем схему $\Sigma_{m}^{\text {unite }}$. Схема $\Sigma_{m}^{\text {unite }}$ содержит $\left(\begin{array}{c}m-1 \\ {[m-1 / 2]}\end{array}\right)$ подсхем, входами которых являются числа нулей $\left(n^{0}\right.$ и $\left.n^{1}\right)$, соответствующие параллельным цепям Анселя.

Выход каждой подсхемы состоит из троичного разряда (mon) и кодов двух новых цепей (или одной, если цепи содержали по одному набору) в кубе размерности $m$. Подсхемы схемы $\Sigma_{m}^{u n i t e}$ осуществляют следующие преобразования:

$$
\begin{aligned}
\operatorname{mon} & =\operatorname{sign}\left(n_{0}-n_{1}\right), \\
n^{l} & =n^{0}+\left(n^{1}=\text { length }\right), \\
n^{s} & =n_{1}-\left(n_{1}=\text { length }\right)
\end{aligned}
$$

Здесь параметр length, число наборов на цепях, есть константа, известная до построения схемы.

Значение $\beta_{i}$ на выходе для $i<m$ определяется таблицей

\begin{tabular}{|c|ccc|}
\hline \multirow{2}{*}{$\beta_{i}^{1}$} & \multicolumn{3}{|c|}{$\beta_{i}^{0}$} \\
\cline { 2 - 4 } & 0 & 1 & - \\
\hline 0 & 0 & $*$ & 0 \\
1 & $*$ & 1 & 1 \\
- & 0 & 1 & - \\
\hline
\end{tabular}

Здесь * означает неполяризуемость функции. Значение $\beta_{m}$ равно -, если все разряды mon равны нулю. Значение $\beta_{m}$ равно 1 , если часть разрядов mon равна 1 , а остальные нулю. Значение $\beta_{m}$ равно 0 , если часть разрядов тоn равна -1 , а остальные - нулю. В остальных случаях $\beta_{m}=*$. Данное преобразование реализуется дополнительной частью схемы $\Sigma_{m}^{\text {unite }}$.

Выходом схемы $\Sigma_{m}^{\text {unite }}$ является конъюнкция по всем $i$ функций $\beta_{i} \neq *$, вектор $\beta$ и набор чисел нулей на новых цепях Анселя ( $n^{l}$ на удлиненных, $n^{s}$ на укороченных).

Лемма 2. Пусть $f\left(x_{1}, \ldots, x_{m-1}, 0\right) u f\left(x_{1}, \ldots, x_{m-1}, 1\right)$ - поляризуемые булевы функции. Тогда СФЭ $\Sigma_{m}^{\text {unite }}$ удовлетворяет условиям теоремы 1 и имеет сложность

$$
\beta(m)=O\left(m^{-1 / 2} 2^{m} \log (m+1)\right) .
$$

Доказательство. Если частичная булева функция неполяризуема, то возможны две ситуации.

1. Есть переменная $x_{i}$, существенная для обеих подфункций, по которой одна из них монотонна, а другая антимонотонна. Тогда для одного вектора поляризации $\beta_{i}=0$, а для другого $\beta_{i}=1$. Это проверяется дополнительной частью схемы $\Sigma_{m}^{\text {unite }}$.

2. Для монотонных функщий

$$
f\left(x_{1}^{\beta_{1}}, \ldots, x_{m-1}^{\beta_{m-1}}, 0\right), \quad f\left(x_{1}^{\beta_{1}}, \ldots, x_{m-1}^{\beta_{m-1}}, 1\right)
$$

функщия $f\left(x_{1}^{\beta_{1}}, \ldots, x_{m-1}^{\beta_{m-1}}, x_{m}\right)$ ни монотонна, ни антимонотонна по $x_{m}$. Последнее возможно лишь, если на одних парах параллельных цепей Анселя нулей больше у $f\left(x_{1}^{\beta_{1}}, \ldots, x_{m-1}^{\beta_{m-1}}, 0\right)$, а на других у $f\left(x_{1}^{\beta_{1}}, \ldots, x_{m-1}^{\beta_{m-1}}, 1\right)$. Это также проверяется дополнительной частью схемы $\Sigma_{m}^{\text {unite }}$.

Код вектора поляризации получается на выходе дополнительной части, код монотонной функшии - на выходах подсхем.

Итак, схема $\Sigma_{m}^{\text {unite }}$ выполняет требуемые в теореме 1 преобразования. Сложность схемы $\Sigma_{m}^{\text {unite }}$ линейна относительно длины ее входа. Лемма доказана. 
С помощью леммы 2 и теоремы 1 полностью аналогично теореме 2 доказывается следующее утверждение.

Теорема 3. Существует последовательность схем $\left\{S_{n}\right\}$, распознающих поляризуемость произвольной функиии п переменных, заданной векторно, сложности $O\left(2^{n} \sqrt{n} \log n\right)$.

\section{4. Частичные линейные функции}

Лемма 3. Пусть частичная функция $f(x)$ доопределима до линейной, $f_{1}(x), f_{2}(x) u f_{3}(x)$ - ее линейные доопределения. Тогда $f_{1}(x) \oplus f_{2}(x) \oplus f_{3}(x)$ - также ее линейное доопределение.

Доказательство. В любой точке, где $f(x)$ определена, выполняются соотношения

$$
f(x)=f_{1}(x)=f_{2}(x)=f_{3}(x)=f_{1}(x) \oplus f_{2}(x) \oplus f_{3}(x) .
$$

Лемма доказана.

Из леммы 3 вытекает важное следствие.

Следствие 2. Множество доопределений частичной линейной функиии образует линейное многообразие.

Кодом частичной линейной булевой функции $f(x)$ назовем совместную систему линейных уравнений

$$
A \alpha=\delta
$$

по модулю 2 верхнетреугольного вида, задающую множество коэффициентов $\alpha$ всевозможных линейных доопределений $f(x)$. Длина кода есть $O\left(m^{2}\right)$. Это кодирование для констант превращается в следующее двухбитное: 00 - неопределенность, 10 - ноль, 11 - единица.

Возможность такого кодирования вытекает из следствия леммы 3.

Лемма 4. Пусть $f_{0}=f\left(x_{1}, \ldots, x_{m-1}, 0\right) u f_{1}=f\left(x_{1}, \ldots, x_{m-1}, 1\right)-$ частичные линейные булевы функции. Тогда существует СФЭ $\Sigma_{m}^{\text {lin }}$, удовлетворяющая условиям теоремы 1 , сложности $\beta(m)=O\left(m^{3}\right)$.

Доказательство. Пусть $A^{0} \alpha=\delta^{0}$ и $A^{1} \alpha=\delta^{1}$ - соответствуюшие системы. Тогда доопределения с фиктивной переменной $x_{m}$ задаются системой

$$
A^{0} \alpha=\delta^{0}, \quad A^{1} \alpha=\delta^{1} .
$$

Доопределения с существенной переменной $x_{m}$ задаются системой

$$
A^{0} \alpha=\delta^{0}, \quad A^{1} \alpha=\delta^{1} \oplus 1 .
$$

Совокупность этих систем объединяется в одну систему

$$
\begin{aligned}
& \left(A^{0} \mid 0\right)\left(\alpha \mid \alpha_{m}\right)=\delta^{0}, \\
& \left(A^{1} \mid 1\right)\left(\alpha \mid \alpha_{m}\right)=\delta^{1} .
\end{aligned}
$$

Схема, приводящая эту систему к верхнетреугольному виду методом Гаусса, имеет сложность $O\left(\mathrm{~m}^{3}\right)$. Лемма доказана. 
Теорема 4. Существует последовательность схем $\left\{S_{n}\right\}$, распознающих доопределимость до линейной произвольной частичной булевой функции п переменных, заданной векторно, сложности $O\left(2^{n}\right)$.

Доказательство. Из теоремы 1 и леммы 4 следует существование схем $S_{n}$, распознающих частичную линейность, сложности

$$
\left(2^{n}-2\right) L\left(x_{1} \& x_{2}\right)+\sum_{k=1}^{n} 2^{n-k} O\left(k^{3}\right) \text {. }
$$

Ряд $\sum_{k=1}^{\infty} k^{3} 2^{-k}$ сходится. По следствию 1 схема $S_{n}$ имеет линейную сложность относительно длины вектор-столбца. Теорема доказана.

В. Б. Алексеевым развит метод логических полуколец (см. [4]), с помощью которого удалось понизить верхние оценки сложности для большого числа задач. Нижняя оценка для этого метода связана с возможностью удачного алгебраического представления исследуемого свойства. В частности, для схемного распознавания монотонных функций, она равнялась $\Omega\left(2^{n} n\right)$. Основным препятствием для удачного применения теоремы 1 является мощность класса функций. Для тех же монотонных функщий, анализируя возможные однозначные кодировки, мы придем к оценке $\Omega\left(2^{n} \sqrt{n}\right)$. Для класса частичных линейных функций это препятствие удалось обойти, используя неоднозначно декодируемую кодировку. Класс частичных линейных функций интересен еще и тем, что к нему [5] сходится сверху счетная цепочка замкнутых классов частичной логики и, следовательно, он не описывается как класс сохранения конечноместного предиката.

\section{Список литературы}

1. Вороненко А. А., О сложности распознавания монотонности. Матем. вопросы киберн. (1999) 8, 301-303.

2. Ансель Ж., О числе монотонных булевых функций $n$ переменных. Киберн. сб. (1968) 5, 42-53.

3. Goldreich O., Goldwasser S., Lehman E., Ron D., Testing monotonicity. Proc. Annual Symp. Found. Computer Sci. (1998), 426-435.

4. Алексеев В. Б., Логические полукольца и их использование для построения быстрых алгоритмов. Вестник МГУ, Серия 1: Математика, механика (1997) №1, 22-29.

5. Алекссев В. Б, Вороненко А. А., О некоторых замкнутых классах в частичной двузначной логике. Дискретная математика (1994) 6, №4, 58-79.

Статья поступила 28.10.2002. 